\title{
VIRTUAL INTELLIGENT SENSORS ON WIDE PLANT CONTROL APPLICATIONS: AN APPROACH
}

\author{
R. Ferreiro García *, X. Pardo Martinez **, F. J. Pérez Castelo *, J Vidal Paz ** \\ Departamento de Ingeniería Industrial *. Departamento de Electrónica y Sistemas** \\ Universidad A Coruña (Spain) \\ ferreiro@udc.es
}

\begin{abstract}
This paper describes a simple and reliable method to acquire information instantaneously on the basis of predictive computations of data acquired with significant time delay. The estimated value of any measured variable is the output of a virtual sensor implemented by means of a virtual engineering programming tool. Copyright (c) 2000 IFAC
\end{abstract}

Keywords: Virtual sensing, Prediction, Filtering, Time-delay, Mapping.

\section{INTRODUCTION}

Transducers are defined as smart or intelligent if they are capable for perform communication tasks between each other by means of digital protocols. The information acquired by several smart sensors can be used to compute any objective or useful variable with the help of some object oriented programming tool. Such computed variable can be regarded as a virtual sensor. The need for any virtual sensor is originally due to difficulty to measure physical magnitudes that can be estimated on the basis of math-models like heat flow, but also to the time lag generated by the primary elements of some sensors like some temperature sensors.

Measurement systems that contain storage elements cannot respond instantaneously to changes in the input. Most primary elements of temperature sensors like thermocouples, resistance temperature detector (RTD), thermistors and solid state temperature sensors are a good example (Tarik Ozkul, 1996).

In practical applications, thermowells separate the temperature measuring sensitive portion of a RTD, thermocouple or filled thermal system, from a potentially corrosive or damaging process media, generating a significant time lag between environment temperature and acquired measure.

The protecting tube in thermocouples and RTD sensors exchange energy with its environment until the two are at same temperature, storing energy during the exchange. The rate at which temperature changes can be modelled as a first order derivative and thermometer behaviour modelled as a first-order equation. In general, systems with a storage or dissipative capability may be modelled using a firstorder differential equation 1.

$$
\begin{aligned}
& F(t)=a_{1} \dot{T}(t)+a_{0} T(t) \\
& \dot{T}(t)=\frac{d T(t)}{d t}
\end{aligned}
$$

Dividing through by a0 gives equation 2 .

$$
\tau \dot{T}(t)+T(t)=K F(t)
$$

which in complex variable domain is equation 3

$$
\frac{T(s)}{T x(s)}=\frac{K}{\tau S+1}
$$


where $\tau=a_{1} / a_{0}$ is called the time constant of the sensor. Regardless of the physical dimensions of $a_{1}$ and $\mathrm{a}_{0}$, their ratio will always have the dimensions of time. The time constant provides a measure of the speed of system response and as such is an important specification in measuring dynamic input signals

\section{MODELLING TEMPERATURE SENSORS WITH PROTECTING TUBE}

According first law of thermodynamics, the rate at which energy is exchanged between the sensor and its environment through convection, Q, must be balanced by the storage of energy within the protecting tube of the sensor, $\mathrm{dE} / \mathrm{dt}$. This conservation of energy is written as equation 4

$$
\frac{d E}{d t}=\dot{Q}
$$

Energy stored in the protecting tube metal is manifested by a change in metal temperature so that for a constant mass, is derived the equation 5

$$
\frac{d E}{d t}=m \cdot C v \cdot \frac{d T(t)}{d t}
$$

Energy exchange by convection between the protecting tube metal at $\mathrm{T}(\mathrm{t})$ and an environment at $\operatorname{Tx}(\mathrm{t})$ according figure 1 , has the form of equation 6.

$$
\begin{aligned}
& \dot{Q}=H \cdot a \cdot \Delta T= \\
& \text { h.A. }[T x(t)-T(t)]
\end{aligned}
$$

The first law can be written as equation 7 .

$$
\begin{aligned}
& \text { m.Cv } \frac{d T(t)}{d t}=\dot{Q}=H \cdot a \cdot \Delta T= \\
& =\text { h.A. }[T x(t)-T(t)]
\end{aligned}
$$

and yields finally in equations 8 and 9.

$$
\frac{m . C v}{h \cdot A} \frac{d T(t)}{d t}+T(t)=T x(t)
$$

or

$$
\tau \frac{d T(t)}{d t}+T(t)=T x(t)
$$

where $m$ is the mass of metal that conforms the protecting tube, $C v$ is the specific heat of tube metal, $h$ is the convection heat transfer coefficient between protecting tube and environment and $A$ is the equivalent concept of the protecting tube surface area.

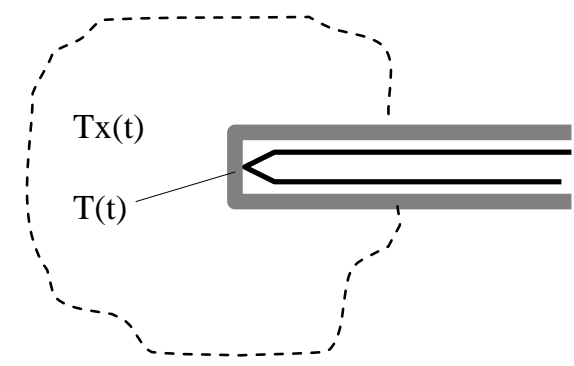

Fig.1. The protecting tube of thermocouple and its environment

In the general case of a forced convection flow, the math-model is similar with time-varying parameters according flow physical conditions.

\section{MODEL BASED PREDICTION OF MEASURED VARIABLE}

Model based prediction depends strongly on modelling errors. In the above model the convection heat transfer coefficient is a relevant fuzzy time varying parameter which depends on physical and chemical properties of the product that conforms the environment temperature. The same product or fluid in liquid or vapour state or at a temperature near boiling point has a different convection heat transfer coefficient and consequently a different model time constant. In general, flow rate and specific heat exerts a strong influence on heat transfer coefficient and consequently on the time constant.

For practical applications it is useful to get an experimental function to represent the time constant as function of fluid flow rate and the category o fluid (liquid or gas specified by its specific heat). Thus the time constant as function of $C V$ and $V$ can be expressed as equation (10).

$$
\tau=f(C v, V)
$$

and the dynamic model as equation 11.

$$
f(C v, V) \frac{d T(t)}{d t}+T(t)=T x(t)
$$

where $C v$ is the specific heat and $V$ is its velocity around the sensor vicinity. As the time constant $\tau$ is expressed as function of $\mathrm{Cv}$ and $V$, the fluid velocity must be measured or estimated to update the time constant, which implies some computation effort. Instead of fluid velocity, sometimes it will be useful to compute the time constant as function of fluid flow for any particular plant. 
In order to update the time constant with no so much time consuming to predict the actual value of the variable to be measured, it is necessary to detect changes in variables related with the measured variable and then, identify the actual model parameters, if any significant change in its actual value has been detected. So that prediction of measured variable is carried out with the help of the actual math-model. This method is useful when the time necessary to detect changes in the measured variable and the time to calculate the time constant is short compared with the value of the time constant. For instance, if the value of time constant is three times larger than the time required to perform the predictive computations, then this operation is well justified.

As a rule of thumb, all signals should be filtered before they are connected to the data acquisition hardware. Some sensors generate not only the primary signal but also high frequency harmonics of the primary signal, which debilitates the data acquisition process.

The signal should be filtered using a filter, which has cut-off frequency twice as high as the highest frequency content of the primary signal. In figure 2 it is shown the general scheme used in this contribution

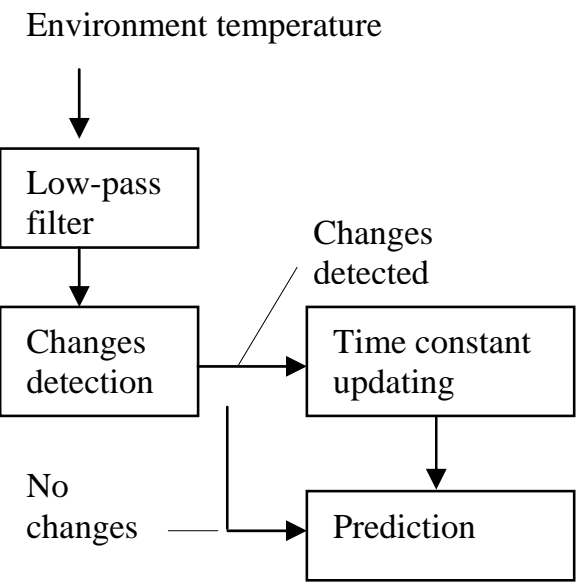

Fig. 2. Diagram of the prediction task

\section{ACHIEVING AN OFF-LINE TIME CONSTANT}

Let's assume the temperature sensor model defined under a linear first order differential equation of equation 12.

$$
\dot{T}=\frac{K}{\tau} T x-\frac{1}{\tau} T
$$

where $\mathrm{T}$ is the actual measured temperature and $\mathrm{Tx}$ is the actual known exciting temperature. The model parameter estimation task is performed by means of a rapid training/learning algorithm, which consists in mapping the sensor dynamics with the real time data shown in the above expression.

Mapping the sensor dynamics requires a data structure of the form shown in figure 3

(a)

(b)

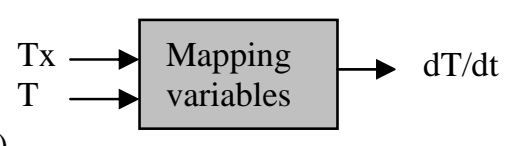

\begin{tabular}{|l|l|l|l|}
\hline Tx\T & T1 & T2 & T3 \\
\hline Tx1 & dT/dt &.. &.. \\
\hline Tx2 &.. &.. &.. \\
\hline Tx3 &.. &.. &.. \\
\hline
\end{tabular}

Fig. 3. Data structure in mapping procedure. (a) Input/output data. (b) Matrix data structure.

Once data has been acquired in an off-line task, it will be immediately processed to achieve the model parameters (mainly the time constant) as equation 13.

$$
\left|\begin{array}{l}
K / \tau \\
1 / \tau
\end{array}\right|=\left[\begin{array}{cc}
T x 1 & T 1 \\
T x 2 & T 2
\end{array}\right]^{-1}\left|\begin{array}{l}
\dot{T} 1 \\
\dot{T} 2
\end{array}\right|
$$

Above task has been performed at a constant fixed fluid flow velocity, and specific heat coefficient. So that, the learning task will be repeated for different fluid velocities and specific heat coefficients (liquid and vapour for instance). The final result is a set of parameters (constant times) which correspond to specific fluid velocities and specific heat coefficients. Processing such data by a polynomial regression gives equation 14

$$
\begin{aligned}
& \tau=C_{0}+C_{1} V+C_{2} V^{2}+C_{3} C v+ \\
& C_{4} C v^{2}+C_{5} V C v+C_{6} V^{2} C v+ \\
& C_{7} V C v^{2}+C_{8} V^{2} C v^{2}
\end{aligned}
$$

Time constant will be computed on-line every time a change in flow velocity or specific heat coefficient had been detected.

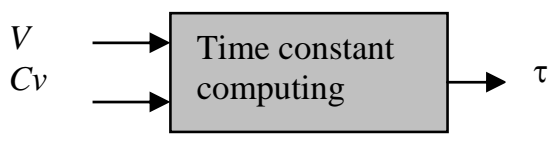

Fig. 4. Input/output data to compute the time constant

\section{VIRTUAL TEMPERATURE SENSOR IMPLEMENTATION}

Implementation of the proposed virtual temperature sensor requires to measure the fluid velocity, its 
specific heat coefficient and its temperature by a smart or conventional sensor.

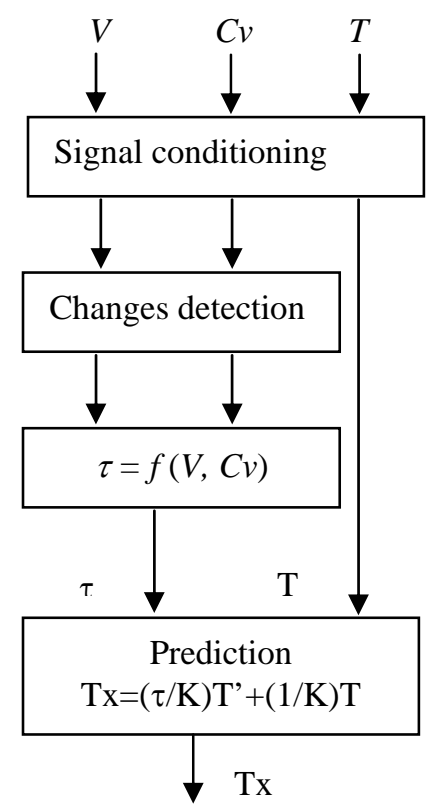

Fig. 5. Structure of a virtual temperature sensor

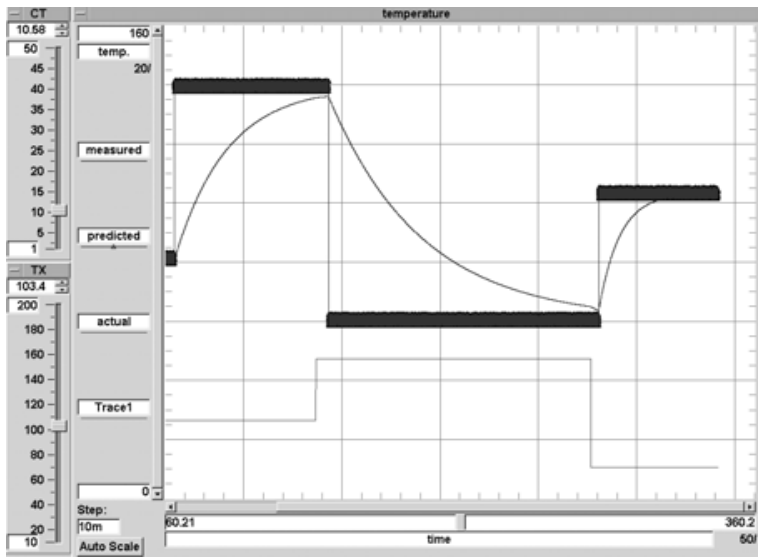

Fig. 6. The virtual sensor response

Once such data is acquired and conditioned, then is processed according the scheme shown in figure 5 . Figure 6 shows the graphical results achieved by computation carried out to predict a temperature sensor output by means of the object oriented programming tool HP-Vee developed by HP (Robert Helsel, 1997)

\section{VIRTUAL HEAT SENSORS ON A HEAT CONTROL LOOP}

In this section it is described a typical application of a virtual heat sensor (William L. Luyben, 1989). Let us consider the problem of controlling the temperature of the fluid in a distillation column where significant pressure changes occur, and it is necessary to adopt a control strategy which consist in applying the computed heat flow as a manipulated variable of the control loop as shown in figure 5. The differential temperature in a heat exchanger is used to infer the heat supplied to the distillation column. Temperature takes some time referred as the time constant of the temperature sensor depending on its primary element characteristics (typically $0.5 \mathrm{sec}$. for thermocouples, 2 sec. for RTD's and 8 sec. for nitrogen filled bulbs into circulating water at $1 \mathrm{~m} / \mathrm{sec}$. velocity).

In gas, vapour or steam this time constants are much greater, sometimes four times greater. So that, in order to achieve precision in measuring values, a strong effort to update temperature values is well justified.

Figure 7 shows the temperature control loop in a distillation column where the manipulated variable is the amount of heat computed as function of fluid flow, and the temperature difference between input and output heating fluid. If heating fluid is oil, the time constant of such sensors is short, but the distillation column temperature sensor might be inserted into a vapour environment, so that time constant is of significant value. The consequence is a time lag introduced by the sensor, which affect the plant control stability and the control performance.

Smart sensors could be used to implement the proposed temperature control loop in which computations to predict the actual value of measured environment would be performed by the proposed virtual sensor. So that, temperature sensors are only used here to measure the temperature with the natural time delay inherent to its characteristics and prediction is performed by the virtual sensor, which is implemented by software under object, oriented programming tools.

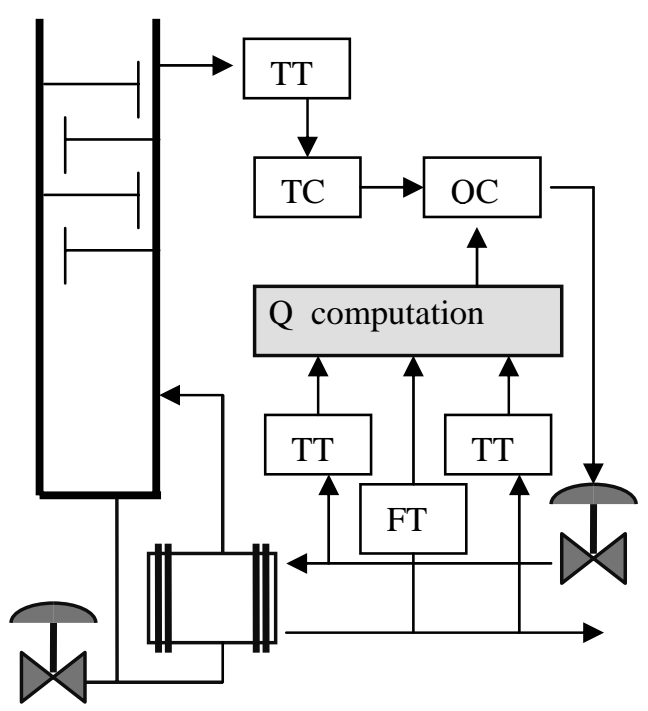

Fig.7. Binary distillation column and temperature control loop 


\section{CONCLUSIONS}

In this work, a general guide to predict the value of a measuring environment when significant time lag in measuring task exist, was described. When fluid states are well known computations of time constant might be simplified. The advances in virtual engineering programming tools bring the facilities to implement any virtual instrument at low cost or effort mainly due to the facility for implement multitask computations. In critical situations where fast changes in the environment exist and must be known without delay, the virtual measuring system improves in the general case of temperatures of liquids at low flow rates or gases.

\section{REFERENCES}

Robert Helsel. (1997). Visual programming with HP VEE. Hewlett-Packard Company. Ed. Prentice Hall PTR. USA.

Tarik Ozkul (1996). Data Acquisition and Process Control Using personal Computers. Pp (102, 459-480)

William L. Luyben (1989). Process Modelling, Simulation and Control for Chemical Engineers.pp(258-259) 